\title{
Text classification using similarity measures on intuitionistic fuzzy sets
}

\author{
Peerasak Intarapaiboon \\ Department of Mathematics and Statistics, Faculty of Science and Technology, Thammasat University, \\ Pathum Thani 12121 Thailand
}

e-mail: peerasak@mathstat.sci.tu.ac.th

Received 14 Jul 2014

Accepted 16 Aug 2015

\begin{abstract}
An intuitionistic fuzzy set (IFS) is an extended version of a fuzzy set and is capable of representing hesitancy degrees. A framework for text classification is presented. Two main challenges are addressed: how to represent documents in terms of IFSs and how to obtain a pattern of each class from such an IFS-based representation. By using some existing similarity measures for IFSs, the proposed framework is applied to two benchmark datasets for text classification. The proposed framework yields satisfactory results when compared to decision tree, $k$-NN, naïve Bayes, and support vector machine classifiers.
\end{abstract}

KEYWORDS: uncertainty representation, text mining, pattern learning

\section{INTRODUCTION}

After the theory of fuzzy sets was proposed by Zadeh $^{1}$, many studies have indicated that the theory facilitates solving various real-world problems, especially when dealing with vague information. In fuzzy set theory, membership and nonmembership degrees are complementary, i.e., the sum of both degrees of an element belonging in a fuzzy set is 1. However, there are some situations that the two degrees are not complementary, mainly because of hesitation. Intuitionistic fuzzy set (IFS) was introduced by Atanassov ${ }^{2}$ to handle such situations. For representing an IFS, each element is assigned by membership and nonmembership degrees, where the sum of the two degrees does not exceed 1. An IFS is therefore more meaningful than a fuzzy set.

Dengfeng and Chuntian ${ }^{3}$ gave the axiomatic definition of similarity measures between IFSs and proposed similarity measures based on high membership and low membership functions. They also paved the way for applying IFS similarity measures to pattern recognition. Liang and $\mathrm{Shi}^{4}$ showed some counter-intuitive cases obtained from the measures proposed in Ref. 3 and then presented several similarity measures to overcome those cases. Mitchell ${ }^{5}$ claimed that the rationale behind unreasonable cases was the weakness of the definition for similarity measures. Thus a stronger definition for grading similarity degree between IFSs was defined. Hung and Yang ${ }^{6}$ adopted the Hausdorff distance for developing several similarity measures. $\mathrm{Xu}^{7}$ introduced the concepts of positive and negative ideal IFSs and extended some similarity measures by assigning weights. The proposed measures were applied to solve multi-attribute decision making problems. Khatibi and Montazer ${ }^{8}$ conducted experiments for bacterial classification using a Euclideanbased measure on fuzzy sets, a Euclidean-based measure on IFSs, and a Hausdorff-based measure on IFSs. The results indicated that the both measures on IFSs outperformed others on fuzzy sets. Most similarity measures in the literature are derived from distance measures. As an alternative way, cosine and weighted cosine similarity measures ${ }^{9}$ for IFSs were first proposed and applied to a small medical diagnosis problem; after that these measures were modified to satisfy the similarity definition by Hwang and Yang ${ }^{10}$. Reviews of similarity measures for IFSs are presented in Refs. 11, 12.

Text categorization, which involves assigning a textual document to a predefined set of categories, is attracting more attention from researchers. Since this task can be seen as a classification problem from a machine learning point of view, several frameworks using a variety of classification techniques have been proposed. Most classification techniques aim to make a pattern for each category. A new document is then assigned to the category such that its pattern is the most similar to the document. Reviews of text categorization can be found in, e.g., Refs. 13-15. There is little on applying IFSs to 
Table 1 Some similarity measures between IFSs.

\begin{tabular}{ll}
\hline Author & Expression \\
\hline Dengfeng $^{3}$ & $S_{d}^{p}(A, B)=1-(1 / \sqrt[p]{h}) \sqrt[p]{\sum_{i=1}^{h}\left|\varphi_{A}(i)-\varphi_{B}(i)\right|^{p}}$ \\
& where $\varphi_{k}(i)=\left(\mu_{k}\left(x_{i}\right)+1-v_{k}\left(x_{i}\right)\right) / 2, k=\{A, B\}$, and $1 \leqslant p \leqslant \infty$ \\
Liang $^{4}$ & $S_{\mathrm{e}}^{p}(A, B)=1-(1 / \sqrt[p]{h}) \sqrt[p]{\sum_{i=1}^{h}\left(\frac{1}{2}\left(\left|\mu_{A}\left(x_{i}\right)-\mu_{B}\left(x_{i}\right)\right|+\left|v_{A}\left(x_{i}\right)-v_{B}\left(x_{i}\right)\right|\right)\right)^{p}}$ \\
Mitchell $^{5}$ & $S_{m}^{p}(A, B)=\frac{1}{2}\left(\rho_{\mu}(A, B)+\rho_{\mathrm{f}}(A, B)\right)$ \\
& where $\rho_{\mu}(A, B)=S_{d}^{p}\left(\mu_{A}\left(x_{i}\right), \mu_{B}\left(x_{i}\right)\right)$ and $\rho_{\mathrm{f}}(A, B)=S_{d}^{p}\left(1-v_{A}\left(x_{i}\right), 1-v_{B}\left(x_{i}\right)\right)$ \\
Xu $^{7}$ & $S_{\mathrm{Z}}(A, B)=1-\left[(1 / 2 h) \sum_{i=1}^{h}\left(\left(\mu_{A}\left(x_{i}\right)-\mu_{B}\left(x_{i}\right)\right)^{p}+\left(v_{A}\left(x_{i}\right)-v_{B}\left(x_{i}\right)\right)^{p}+\left(\pi_{A}\left(x_{i}\right)-\pi_{B}\left(x_{i}\right)\right)^{p}\right)\right]^{1 / p}$ \\
Julian $^{17}$ & $S_{n}^{p}(A, B)=1-\sqrt[p]{\sum_{i=1}^{h} w_{i}\left(\left|\mu_{A}\left(x_{i}\right)-\mu_{B}\left(x_{i}\right)\right|\right)^{p}}-\sqrt[p]{\sum_{i=1}^{h} w_{i}\left(\left|v_{A}\left(x_{i}\right)-v_{B}\left(x_{i}\right)\right|\right)^{p}}$ \\
& with $w_{i} \geqslant 0$ and $\sum_{i=1}^{h} w_{i}=1$ and $p \geqslant 1$ \\
Ye $^{9}$ & $S_{C}(A, B)=(1 / h) \sum_{i=1}^{h}\left(\mu_{A}\left(x_{i}\right) \mu_{B}\left(x_{i}\right)+v_{A}\left(x_{i}\right) v_{B}\left(x_{i}\right)\right) /\left(\sqrt{\mu_{A}^{2}\left(x_{i}\right)+v_{A}^{2}\left(x_{i}\right)} \sqrt{\mu_{B}^{2}\left(x_{i}\right)+v_{B}^{2}\left(x_{i}\right)}\right)$ \\
Hwang and Yang $^{10}$ & $S_{S}(A, B)=\frac{1}{3}\left(S_{C}(A, B)+C_{\mathrm{IFS}}^{*}(A, B)+C_{\mathrm{IFS}}^{* *}(A, B)\right)$ \\
& where $C_{\mathrm{IFS}}^{*}(A, B)=\frac{1}{h} \sum_{i=1}^{h} \frac{\varphi_{A}\left(x_{i}\right) \varphi_{B}\left(x_{i}\right)+v_{A}\left(x_{i}\right) v_{B}\left(x_{i}\right)}{\sqrt{\varphi_{A}^{2}\left(x_{i}\right)+v_{A}^{2}\left(x_{i}\right)} \sqrt{\varphi_{B}^{2}\left(x_{i}\right)+v_{B}^{2}\left(x_{i}\right)}}$ \\
& with $\varphi_{k}\left(x_{i}\right)=\frac{1}{2}\left(1+v_{k}\left(x_{i}\right)-v_{k}\left(x_{i}\right)\right), k=A, B$ and \\
& $C_{\mathrm{IFS}}^{* *}(A, B)=\frac{1}{h} \sum_{i=1}^{h} \frac{\left(1-\mu_{A}\left(x_{i}\right)\right)\left(1-\mu_{B}\left(x_{i}\right)\right)+\left(1-v_{A}\left(x_{i}\right)\right)\left(1-v_{B}\left(x_{i}\right)\right)}{\sqrt{\left(1-\mu_{A}\left(x_{i}\right)\right)^{2}+\left(1-v_{A}\left(x_{i}\right)\right)^{2}} \sqrt{\left(1-\mu_{B}\left(x_{i}\right)\right)^{2}+\left(1-v_{B}\left(x_{i}\right)\right)^{2}}}$ \\
&
\end{tabular}

text categorization in the literature. Szmidt and Kacprzyk ${ }^{16}$ proposed a strategy for feature selection in text categorization using the concept of IFSs. No framework obviously shows the benefit of IFS similarity measures to text categorization.

To shed light on this study direction, a framework for text classification based on IFS similarity measures is presented. We address how to represent a document in terms of an IFS and how to obtain a pattern of each class from such an IFS-based representation. Our framework is then evaluated on two benchmark datasets of text classification and compared to other traditional text classification methods.

\section{INTUITIONISTIC FUZZY SETS AND THEIR SIMILARITY MEASURES}

In this section, we present some basic concepts for IFSs and their similarity measures. The following notation is used hereinafter: $X=\left\{x_{1}, x_{2}, \ldots, x_{h}\right\}$ is a discrete universe of discourse; $\operatorname{IFS}(X)$ is the collection of all IFSs of $X$. An intuitionistic fuzzy set $A$ in $\operatorname{IFS}(X)$ is defined as follows:

$$
A=\left\{\left\langle x_{i}, \mu_{A}\left(x_{i}\right), v_{A}\left(x_{i}\right)\right\rangle \mid x_{i} \in X\right\}
$$

which is characterized by a membership function $\mu_{A}\left(x_{i}\right)$ and a nonmembership function $v_{A}\left(x_{i}\right)$. The two functions are defined as follows:

$$
\mu_{A}: X \rightarrow[0,1],
$$

$$
v_{A}: X \rightarrow[0,1]
$$

such that

$$
0 \leqslant \mu_{A}\left(x_{i}\right)+v_{A}\left(x_{i}\right) \leqslant 1, \quad \forall x_{i} \in X .
$$

Another degree, $\pi_{A}\left(x_{i}\right)$, the hesitancy degree of $x_{i}$ belonging to $A$, is defined as

$$
\pi_{A}\left(x_{i}\right)=1-\mu_{A}\left(x_{i}\right)-v_{A}\left(x_{i}\right) .
$$

Definition 1 Let $S$ be a real-valued function such that $S: \operatorname{IFS}(X) \times \operatorname{IFS}(X) \rightarrow[0,1] . \quad S$ is called a similarity measure if, for all $A, B, C$ in $\operatorname{IFS}(X)$, it satisfies the following conditions: $S(A, B)=S(B, A)$; $S(A, B)=1$ iff $A=B$; if $A \subseteq B \subseteq C$, then $S(A, C) \leqslant$ $S(A, B)$ and $S(A, C) \leqslant S(B, C)$.

Assuming $A=\left\{\left\langle x_{i}, \mu_{A}\left(x_{i}\right), v_{A}\left(x_{i}\right)\right\rangle \mid x_{i} \in X\right\}$ and $B=\left\{\left\langle x_{i}, \mu_{B}\left(x_{i}\right), v_{B}\left(x_{i}\right)\right\rangle \mid x_{i} \in X\right\}$ are IFSs, Table 1 highlights some similarity measures between IFSs. $S_{d}^{p}, S_{\mathrm{e}}^{p}, S_{m}^{p}, S_{\mathrm{Z}}$, and $S_{n}^{p}$ are distance-based measures, since each of them is defined as one minus the distance. $S_{C}$ and $S_{S}$ are cosine-based measures.

\section{AN IFS-BASED FRAMEWORK FOR TEXT CLASSIFICATION}

The proposed IFS-based framework for text classification is outlined in Fig. 1. The details of this framework are discussed below. 


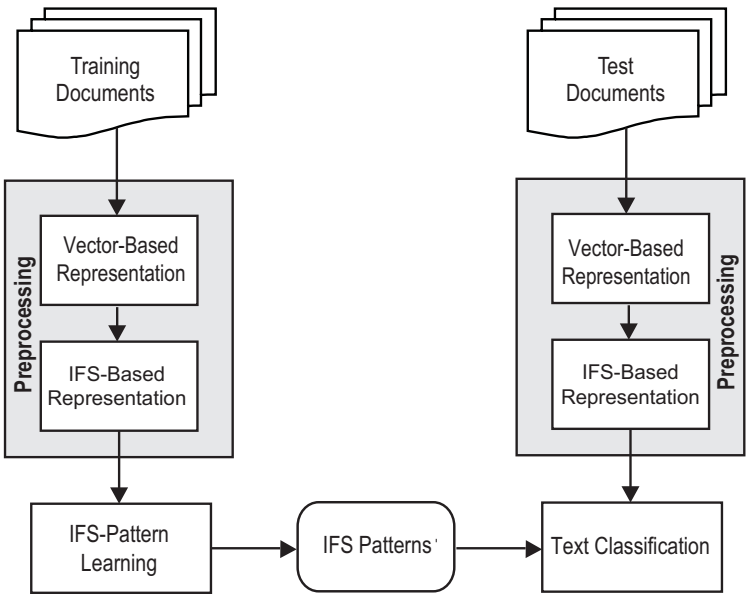

Fig. 1 An overview of the proposed framework.

\section{Preprocessing}

In the preprocessing step, a document is represented as a bag of words, one of the basic methods for representing a document. The bag of words is used to form a vector for representing a document using the frequency of each word determined as a relevant feature for categorization. Such words can be selected by some feature selection techniques, e.g., information gain and gain ratio. Assume $h$ words, i.e., $w_{1}, w_{2}, \ldots, w_{h}$, are selected for this representation. Document $d_{i}$ can be represented by

$$
V_{i}=\left(n_{i, 1}, n_{i, 2}, \ldots, n_{i, h}\right),
$$

where $n_{i, j}$ denotes the number of occurrences of $w_{j}$ in $d_{i}, j=1,2, \ldots, h$.

To convert a bag of words vector to an IFS, we propose one method of which the conceptual idea is explained as follows: Suppose $A_{i}=$ $\left\{\left\langle x_{1}, \mu_{i}\left(x_{1}\right), v_{i}\left(x_{1}\right)\right\rangle \ldots\left\langle x_{h}, \mu_{i}\left(x_{h}\right), v_{i}\left(x_{h}\right)\right\rangle\right\}$ is an IFS for the vector $V_{i}$ in (2). In this work, $\mu_{i}\left(x_{j}\right)$ represents a confidence level to say that word $w_{j}$ occurs in document $d_{i}$, while $v_{i}\left(x_{j}\right)$ is a confidence level to say that word $w_{j}$ does not occur in document $d_{i}$. The next example gives more details.

Example 1 Assume there are three documents, i.e., $d_{1}, d_{2}$, and $d_{3}$, four feature words, i.e., $w_{1}, w_{2}, w_{3}$, and $w_{4}$, and the bag of words-based vectors for these documents, respectively, are

$$
V_{1}=(10,2,5,0), V_{2}=(5,9,5,1), V_{3}=(1,8,4,3) .
$$

Since $n_{1,1}>n_{2,1}>n_{3,1}$, the confidence level to say that $w_{1}$ occurs in $d_{1}$ should be more than for $d_{2}$ and $d_{3}$. It implies that $\mu_{1}\left(x_{1}\right)>\mu_{2}\left(x_{1}\right)>\mu_{3}\left(x_{1}\right)$ and $v_{1}\left(x_{1}\right)<v_{2}\left(x_{1}\right)<v_{3}\left(x_{1}\right)$. Consider $w_{2}$. We should have $\mu_{1}\left(x_{1}\right)<\mu_{2}\left(x_{1}\right) \approx \mu_{3}\left(x_{1}\right)$ and $v_{1}\left(x_{1}\right)>$ $v_{2}\left(x_{1}\right) \approx v_{3}\left(x_{1}\right)$. In the case of $w_{3}$ where $n_{1,3}=$ $n_{2,3} \approx n_{3,3}$, we have low confidence to assign high values for the membership and nonmembership degrees of every document. Thus we set $\mu_{1}\left(x_{3}\right)=$ $\mu_{2}\left(x_{3}\right)=0.2, \mu_{3}\left(x_{3}\right)=0.15, v_{1}\left(x_{3}\right)=v_{2}\left(x_{3}\right)=0.2$, and $v_{3}\left(x_{3}\right)=0.15$.

Based on the ideas discussed above, the process of transformation will be formally explained. Given the universe of discourse $X=\left\{\mathrm{HF}_{1}, \mathrm{HF}_{2}, \ldots, \mathrm{HF}_{h}\right\}$. $\left(\mathrm{HF}_{i}\right.$ has an internal meaning as a high frequency of $w_{i}$.) Every value $n_{i, j}$ in the vector-based representation of the document $i$ is then expressed in terms of the three degrees of $\mathrm{HF}_{j}$ as in the following steps:

(i) $n_{i, j}$ is normalized by

$$
z_{i, j}=\frac{n_{i, j}-\bar{X}_{j}}{s_{j}},
$$

where $\bar{X}_{j}$ and $s_{j}$ are the mean and the standard deviation, respectively, of the feature word $w_{j}$.

(ii) Denoted by $\mu_{i, j}$, a membership degree of the document $i$ with respect to $\mathrm{HF}_{j}$ is determined by a weighted sigmoid function:

$$
\mu_{i, j}=\frac{r_{j}}{1+\mathrm{e}^{-z_{i, j}}},
$$

where $r_{j}$ is a weight for $\mathrm{HF}_{j}$.

(iii) Denoted by $v_{i, j}$, the nonmembership degree of the document $i$ with respect to $\mathrm{HF}_{j}$ is calculated by a weighted sigmoid function:

$$
v_{i, j}=\frac{r_{j}^{*}}{1+\mathrm{e}^{z_{i, j}}},
$$

where $r_{j}^{*}$ is a weight for $\mathrm{HF}_{j}$.

(iv) Denoted by $\pi_{i, j}$, the hesitancy degree of the document $i$ with respect to $\mathrm{HF}_{j}$ is calculated by (1), i.e.,

$$
\pi_{i, j}=1-\mu_{i, j}-v_{i, j} .
$$

\section{Pattern learning}

This section presents a procedure for learning patterns of predefined classes in terms of IFSs. Assume that there are $l$ classes referred to as $C_{1}, C_{2}, \ldots, C_{l}$; $m$ training documents denoted by $d_{1}, d_{2}, \ldots, d_{m}$; and $h$ word features, namely, $w_{1}, w_{2}, \ldots, w_{h}$. A pattern for class $C_{k}$, denoted by $P_{k}$, is defined by

$$
P_{k}=\left\{\left(\mathrm{HF}_{j}, \bar{\mu}_{k j}, \bar{v}_{k j}\right)\right\}_{j=1}^{h},
$$

where $\bar{\mu}_{k j}$ and $\bar{v}_{k j}$ are the average values of membership and nonmembership, respectively, of the 
Table 2 A training set used in Example 2.

\begin{tabular}{lcccc}
\hline Document & $w_{1}$ & $w_{2}$ & $w_{3}$ & Class \\
\hline$d_{1}$ & 15 & 2 & 7 & $C_{1}$ \\
$d_{2}$ & 10 & 3 & 5 & $C_{1}$ \\
$d_{3}$ & 2 & 9 & 6 & $C_{2}$ \\
$d_{4}$ & 3 & 11 & 5 & $C_{2}$ \\
$d_{5}$ & 3 & 9 & 4 & $C_{2}$ \\
\hline
\end{tabular}

word feature $w_{j}$ observed from $d_{i}$ belonging to $C_{k}$. More precisely,

$$
\bar{\mu}_{k j}=\frac{\sum_{i=1}^{m}\left(\mu_{i j} \chi_{k}\left(d_{i}\right)\right)}{\sum_{i=1}^{m} \chi_{k}\left(d_{i}\right)}, \bar{v}_{k j}=\frac{\sum_{i=1}^{m}\left(v_{i j} \chi_{k}\left(d_{i}\right)\right)}{\sum_{i=1}^{m} \chi_{k}\left(d_{i}\right)},
$$

where

$$
\chi_{k}\left(d_{i}\right)= \begin{cases}1, & d_{i} \in C_{k} \\ 0, & \text { otherwise }\end{cases}
$$

\section{Text classification}

To assign a proper class to a test document $d_{t}$, we represent $d_{t}$ in terms of an IFS by the same values of parameters during the training process. Intuitively, $d_{t}$ is grouped into class $C^{\prime}$ such that its pattern is closest to the IFS representation of $d_{t}$. More formally,

$$
C^{\prime}=\arg \max _{C_{k}}\left\{\operatorname{Sim}\left(P_{k}, \operatorname{IFS}_{d_{t}}\right)\right\},
$$

where Sim is a similarity measure between IFSs; $P_{k}$ is an IFS-based pattern of class $C_{k}$; and IFS $d_{t}$ is the IFS representation of $d_{t}$.

Example 2 This example shows the details of the proposed framework. Assume that there are five training documents, i.e., $d_{1}, d_{2}, d_{3}, d_{4}$, and $d_{5}$; three feature words, i.e., $w_{1}, w_{2}$, and $w_{3}$; and two document classes, referred to as $C_{1}$ and $C_{2}$. The frequency of each word and the class of each document are depicted in Table 2. For example, $d_{1}$ has 15,2 , and 7 occurrences of $w_{1}, w_{2}$, and $w_{3}$, respectively, and its class is $C_{1}$.

To represent those documents as IFSs, we start by defining the universe $X=\left\{\mathrm{HF}_{1}, \mathrm{HF}_{2}, \mathrm{HF}_{3}\right\}$. From Table $2, \bar{X}_{1}, \bar{X}_{2}, \bar{X}_{3}, s_{1}, s_{2}$, and $s_{3}$ are 6.6, 6.8, 5.4, $5.68,4.02$, and 1.14 , respectively. By (3), (4), and (5) with $r_{j}=r_{j}^{*}=0.9$, we obtain IFSs corresponding to these documents as

$$
\begin{aligned}
\mathrm{IFS}_{1}= & \left\{\left\langle\mathrm{HF}_{1}, 0.73,0.17\right\rangle,\left\langle\mathrm{HF}_{2}, 0.21,0.69\right\rangle,\right. \\
& \left.\left\langle\mathrm{HF}_{3}, 0.72,0.18\right\rangle\right\}, \\
\mathrm{IFS}_{2}= & \left\{\left\langle\mathrm{HF}_{1}, 0.58,0.32\right\rangle,\left\langle\mathrm{HF}_{2}, 0.25,0.65\right\rangle,\right. \\
& \left.\left\langle\mathrm{HF}_{3}, 0.37,0.53\right\rangle\right\}, \\
\mathrm{IFS}_{3}= & \left\{\left\langle\mathrm{HF}_{1}, 0.28,0.62\right\rangle,\left\langle\mathrm{HF}_{2}, 0.57,0.33\right\rangle,\right. \\
& \left.\left\langle\mathrm{HF}_{3}, 0.57,0.33\right\rangle\right\}, \\
\mathrm{IFS}_{4}=\{ & \left\{\left\langle\mathrm{HF}_{1}, 0.31,0.59\right\rangle,\left\langle\mathrm{HF}_{2}, 0.67,0.23\right\rangle,\right. \\
& \left.\left\langle\mathrm{HF}_{3}, 0.37,0.53\right\rangle\right\}, \\
\mathrm{IFS}_{5}= & \left\{\left\langle\mathrm{HF}_{1}, 0.31,0.59\right\rangle,\left\langle\mathrm{HF}_{2}, 0.57,0.33\right\rangle,\right. \\
& \left.\left\langle\mathrm{HF}_{3}, 0.20,0.70\right\rangle\right\} .
\end{aligned}
$$

To construct class patterns, the documents are grouped by their classes and then the class pattern can be obtained by averaging membership and nonmembership levels. The patterns are

$$
\begin{aligned}
P_{1}= & \left\{\left\langle\mathrm{HF}_{1}, 0.66,0.24\right\rangle,\left\langle\mathrm{HF}_{2}, 0.23,0.67\right\rangle,\right. \\
& \left\langle\mathrm{HF}_{3}, 0.55,0.35\right\rangle,, \\
P_{2}= & \left\{\left\langle\mathrm{HF}_{1}, 0.30,0.60\right\rangle,\left\langle\mathrm{HF}_{2}, 0.60,0.30\right\rangle,\right. \\
& \left.\left\langle\mathrm{HF}_{3}, 0.38,0.52\right\rangle\right\} .
\end{aligned}
$$

Suppose that $d_{t}$ is a new document to be classified in which the frequencies of feature words $w_{1}, w_{2}$, and $w_{3}$ are 10,5 , and 5 , respectively. Using the same values of $\bar{X}_{j}$ and $s_{j}$ in the training process, $d_{t}$ can be represented as an IFS:

$$
\begin{aligned}
\mathrm{IFS}_{d_{t}}= & \left\{\left\langle\mathrm{HF}_{1}, 0.58,0.32\right\rangle,\right. \\
& \left.\left\langle\mathrm{HF}_{2}, 0.39,0.51\right\rangle,\left\langle\mathrm{HF}_{3}, 0.39,0.51\right\rangle\right\} .
\end{aligned}
$$

If $S_{C}$ in Table 1 is used to calculate the similarity between $P_{k}$ and IFS ${ }_{d_{t}}$, where $k=1,2$, then we have

$$
S_{C}\left(P_{1}, \operatorname{IFS}_{d_{t}}\right)=0.96, \quad S_{C}\left(P_{2}, \operatorname{IFS}_{d_{t}}\right)=0.91 .
$$

Hence $d_{t}$ is classified as $C_{1}$.

\section{DATASETS AND EXPERIMENTAL SETTINGS}

\section{Datasets}

In this paper, two news datasets ${ }^{18}$, namely, BBC and BBCSport, were used for our experiments. The BBC dataset is constructed from 2225 news articles in 5 topical areas. With basic preprocessing steps including stemming, stop-word removal, and low term frequency filtering, 9635 words are obtained for representing each article. The BBCSport dataset contains 737 sports news articles on 5 areas. Each article is represented by the frequency of 4613 words obtained using the same processes applied to BBC. Table 3 summarizes important characteristics of each dataset. 
Table 3 Data set characteristics.

\begin{tabular}{lrcc}
\hline Dataset & \#articles & \#words & Class distribution \\
\hline BBC & 2225 & 9635 & $474: 374: 434: 506: 437$ \\
BBCSport & 737 & 4613 & $101: 124: 265: 147: 100$ \\
\hline
\end{tabular}

\section{Experimental schema and parameter setting}

As seen in Table 3, the feature-space dimensions of both datasets are very high. In order to reduce the dimensions, two measures, the gain ratio (GR) and information gain (IG) were applied for evaluating feature relevance. The top $20 \%$ and $30 \%$ of features ranked according to their GR and IG values for BBC and the top $50 \%$ and $80 \%$ of those for BBCSport were selected.

To retain the independence of the data in use, 5 -fold cross-validation (CV) was adopted for evaluating the proposed framework. In our experiment, we explore two strategies for setting the weights $r_{j}$ and $r_{j}^{*}$ in (4) and (5):

(i) A varied grid-based method, referred to as VG, varies the weights from $0.6-1$ with increments of 0.1 . If 0.8 and 0.9 are set for $r_{j}$ and $r_{j}^{*}$, respectively, then $r_{j}$ is fixed at 0.8 while $r_{j}^{*}$ is 0.9 for all $j$. There are thus 25 alternatives which come from the combinations of $0.6-1$ of $r_{j}$ and $0.6-1$ of $r_{j}^{*}$.

(ii) An adaptive method, referred to as $\mathrm{AD}$, sets the two weights based on statistical characteristics of feature words by

$$
r_{j}=r_{j}^{*}=\left|\frac{1-s_{j}}{1+s_{j}}\right|,
$$

where $s_{j}$ is the standard deviation of the number of occurrences of $w_{j}$ in training documents.

In this paper, all IFS similarity measures listed in Table 1 were used when the parameter $p$ was set to 2 for $S_{d}^{p}, S_{\mathrm{e}}^{p}, S_{m}^{p}, S_{\mathrm{Z}}$, and $S_{n}^{p}$, and each $w_{i}$ of $S_{n}^{p}$ was set equal to the reciprocal of the number of features.

\section{Evaluation metric}

Given a test set, $l$ denotes the number of classes, and $n_{i}$ denotes the number of documents in class $i$. When an experiment is done, some preliminary performance measures with respect to class $i$, i.e., precision $\left(P_{i}\right)$, recall $\left(R_{i}\right)$, and F-measure $\left(F_{i}\right)$, are calculated. They are defined by

$$
P_{i}=\frac{\mathrm{TP}_{i}}{\mathrm{TP}_{i}+\mathrm{FP}_{i}}, R_{i}=\frac{\mathrm{TP}_{i}}{n_{i}}, F_{i}=\frac{2 R_{i} P_{i}}{R_{i}+P_{i}},
$$

where $\mathrm{TP}_{i}$ (true positive) refers to the number of documents correctly classified as class $i$; with $i \neq$ $j, F N_{i}$ (false negative) refers to the number of documents in class $i$ classified as class $j$; and $\mathrm{FP}_{i}$ (false positive) to the number of documents in $j$ classified as $i$. Since every subdataset for the 5 -fold CV contains 5 classes and the numbers of documents belonging in the classes are not equal, all experiments are multi-class imbalance scenarios. To show the average performance over all classes, we use the mean F-measure (MFM) and macro geometric average $(\mathrm{MAvG})$, which are widely used in this situation ${ }^{19}$, were applied. These two evaluation metrics are defined as follows:

$$
\mathrm{MFM}=\frac{\sum_{i=1}^{l} F_{i}}{l}, \quad \mathrm{MAvG}=\sqrt[l]{\prod_{i=1}^{l} R_{i}} .
$$

To present a level of false classification for the proposed framework, AvD, the average difference between the similarity degree with the correct classes and that with the predicted classes over all false predictions, is calculated as

$$
\begin{aligned}
\operatorname{AvD}=\sum_{d_{t} \in \mathrm{FC}}\left(\mid \operatorname{Sim}\left(\operatorname{IFS}\left(d_{t}\right), \operatorname{IFS}\left(\operatorname{Cor}_{d_{t}}\right)\right)\right. \\
\left.\quad-\operatorname{Sim}\left(\operatorname{IFS}\left(d_{t}\right), \operatorname{IFS}\left(\operatorname{Prt}_{d_{t}}\right)\right) \mid\right) /|\mathrm{FC}|,
\end{aligned}
$$

where $d_{t}$ is a test document, IFS $\left(d_{t}\right)$ is the IFS representing $d_{t}$, IFS $\left(\mathrm{Cor}_{d_{t}}\right)$ is the IFS representing the correct class of $d_{t}$, IFS $\left(\operatorname{Prt}_{d_{t}}\right)$ is the IFS representing the predicted class of $d_{t}$, and FC is the set of false classified documents.

\section{EXPERIMENTAL RESULTS AND DISCUSSION}

The first experiment aims to compare performances of the varied grid-based and adaptive strategies for weight setting. The second experiment compares our IFS-based framework with traditional classification methods including decision tree, $k$-NN, naïve Bayes, and support vector machine.

\section{Comparison between the VG and AD weight setting methods}

Using the similarity measures in Table 1 , Tables 4 and 5 give the evaluation results on BBC when the gain ratio and information gain are used, respectively. Likewise, Tables 6 and 7 give results on BBCSport. In each table, the first column presents the percentage of selected features, the second means the strategy of weight setting, and the others show experimental results in terms of MFM (in percent), MAvG (in percent), and AvD. A value in parentheses after an evaluation score presents the uncertainty of the last figure in terms of the standard deviation 
Table 4 Comparison between the VG and $\mathrm{AD}$ methods on BBC with top $N \%$ of features ranked by GR.

\begin{tabular}{|c|c|c|c|c|c|}
\hline$N$ & Sim & W & MFM(SD) & MAvG(SD) & $\mathrm{AvD}(\mathrm{SD})$ \\
\hline \multirow[t]{14}{*}{20} & \multirow[t]{2}{*}{$S_{d}^{p}$} & $\mathrm{AD}$ & $93.2(4)$ & 9 & 10 \\
\hline & & VG & $94.3(9)^{[1}$ & & $-4(4)^{[0 .}$ \\
\hline & \multirow[t]{2}{*}{$S_{\mathrm{e}}^{p}$} & $\mathrm{AD}$ & $93.2(4)$ & 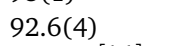 & $-4(5) \uparrow$ \\
\hline & & VG & 94.3 & & ${ }^{-4}(4)^{[}$ \\
\hline & \multirow[t]{2}{*}{$S_{m}^{p}$} & $\mathrm{AD}$ & $93.2(4)$ & & $-4(2) \uparrow$ \\
\hline & & VG & 94. & & $-4(2)^{[\mathrm{C}}$ \\
\hline & \multirow[t]{2}{*}{$S_{z}$} & $\mathrm{AD}$ & 93.2 & & $-4(5) \uparrow$ \\
\hline & & VG & $94.3(9)^{[1,1]}$ & $93.9(1)^{[1,1]}$ & $0^{-4}(4)^{[0.6,0.6]}$ \\
\hline & \multirow[t]{2}{*}{$S_{n}^{p}$} & $\mathrm{AD}$ & 93.2 & 92.6 & $-4(1)$ \\
\hline & & VG & & & $-3(1)^{[4}$ \\
\hline & \multirow[t]{2}{*}{$S_{C}$} & $\mathrm{AD}$ & 92.8 & 92.1 & ${ }^{-4}(1)$ \\
\hline & & VG & & & ${ }^{-4}(7)^{[}$ \\
\hline & \multirow[t]{2}{*}{$S_{S}$} & $\mathrm{AD}$ & 85. & 82 & 3. \\
\hline & & VG & 91. & 89 & ${ }^{-5}(6)$ \\
\hline \multirow[t]{14}{*}{30} & \multirow[t]{2}{*}{$S_{d}^{p}$} & $\mathrm{AD}$ & 94.7 & 9 & $-4(3) \uparrow$ \\
\hline & & VG & & & ${ }^{4}(6)^{[\mathrm{C}}$ \\
\hline & & $\mathrm{AD}$ & 94.7 & & $-4(3)$ \\
\hline & & VG & 94. & & $-4(6)^{[}$ \\
\hline & \multirow[t]{2}{*}{$S_{m}^{p}$} & $\mathrm{AD}$ & 94.7( & 94.4 & $1.310^{-4}(1)$ \\
\hline & & VG & & & )$^{-4}(3)^{[0 .}$ \\
\hline & & $\mathrm{AD}$ & 94. & 94 & $0^{-4}(3)$ \\
\hline & & VG & & & $-4(6)^{[0.6,}$ \\
\hline & \multirow[t]{2}{*}{$S_{n}^{p}$} & $\mathrm{AD}$ & $94.7(6$ & 94.4 & $5.210^{-4}(7) \uparrow$ \\
\hline & & VG & 94.7 & & 1 \\
\hline & \multirow[t]{2}{*}{$S_{C}$} & $\mathrm{AD}$ & $93.4(1)$ & $93.0(1$ & $2.210^{-4}(1)$ \\
\hline & & VG & & & 1.71 \\
\hline & \multirow[t]{2}{*}{$S_{S}$} & $\mathrm{AD}$ & $84.8(2)$ & $82.8(2)$ & $6.010^{-5}(7)$ \\
\hline & & VG & & & \\
\hline
\end{tabular}

(SD). For instance, 93.2(4) means $93.2 \pm 0.4$, while $3.310^{-4}(5)$ means $0.00033 \pm 0.00005$.

For brevity, only the best value of each metric is reported for the VG method. The superscripts $[x, y]$ denote the values of $r_{j}$ and $r_{j}^{*}$, respectively, that yield such the highest value.

From the tables, we can see that the experimental results from $\mathrm{AD}$ are comparable to those from VG. For the tests on BBC using gain ratio for feature selection (Table 4), regardless of similarity measures, the MFM and MAvG scores resulting from our framework with VG are slightly higher than those from our framework with AD. In contrast, considering the AvD values, the framework with $\mathrm{AD}$ outperforms the framework with VG with the average difference of $3.2010^{-4}$. With information gain (Table 5), all the results obtained from the distance-based measures, i.e., $S_{d}^{p}, S_{\mathrm{e}}^{p}, S_{m}^{p}, S_{z}$, and $S_{n}^{p}$ with $\mathrm{AD}$ are better than those with VG. The cosinebased measures, i.e., $S_{C}$ and $S_{S}$, with $\mathrm{AD}$ yield lower values of MFM and MAvG than the same measures with VG. Likewise, on BBCSport (Tables 6 and 7), we observe that the experimental results from $\mathrm{AD}$ are comparable to those from VG.
Table 5 Comparison between the VG and AD methods on BBC with top $N \%$ of features ranked by IG.

\begin{tabular}{|c|c|c|c|c|c|}
\hline$N$ & Sim & $\mathrm{W}$ & MFM(SD) & MAvG(SD) & $\mathrm{AvD}(\mathrm{SD})$ \\
\hline \multirow[t]{14}{*}{20} & \multirow[t]{2}{*}{$S_{d}^{p}$} & $\mathrm{AD}$ & $94.3(9)$ & $94.0(1)$ & $3.610^{-4}(5) \uparrow$ \\
\hline & & VG & $93.9(1)^{[1,1]}$ & $93.6(1)^{[1,1]}$ & $7.110^{-4}(1)^{[0.6,0.6]}$ \\
\hline & \multirow[t]{2}{*}{$S_{\mathrm{e}}^{p}$} & $\mathrm{AD}$ & $94.4(9)$ & $94.0(1)$ & $3.610^{-4}(5) \uparrow$ \\
\hline & & VG & $93.9(1)^{[1,1]}$ & $93.6(1)^{[1,1]}$ & $7.110^{-4}(1)^{[0.6,0.6]}$ \\
\hline & \multirow[t]{2}{*}{$S_{m}^{p}$} & $\mathrm{AD}$ & $94.4(9)$ & $94.0(1)$ & $1.810^{-4}(3) \uparrow$ \\
\hline & & VG & $93.9(1)^{[1,1]}$ & $93.6(1)^{[1,1]}$ & $3.610^{-4}(6)^{[0.6,0.6]}$ \\
\hline & \multirow[t]{2}{*}{$S_{z}$} & $\mathrm{AD}$ & $94.4(9)$ & $94.0(1)$ & $3.610^{-4}(5) \uparrow$ \\
\hline & & VG & $93.9(1)^{[1,1]}$ & $93.6(1)^{[1,1]}$ & $7.110^{-4}(1)^{[0.6,0.6]}$ \\
\hline & \multirow[t]{2}{*}{$S_{n}^{p}$} & $\mathrm{AD}$ & $94.4(9)$ & $94.0(1)$ & $7.310^{-4}(1) \uparrow$ \\
\hline & & VG & $93.9(1)^{[1,1]}$ & $93.6(1)^{[1,1]}$ & $1.410^{-3}(2)^{[0.6,0.6]}$ \\
\hline & \multirow[t]{2}{*}{$s_{C}$} & $\mathrm{AD}$ & $92.3(2) \downarrow$ & $91.8(2) \downarrow$ & $3.710^{-4}(5)$ \\
\hline & & VG & $94.6(1)^{[0.6,1]}$ & $93.0(1)^{[0.6,1]}$ & $3.010^{-4}(4)^{[1,0.6]}$ \\
\hline & \multirow[t]{2}{*}{$S_{S}$} & $\mathrm{AD}$ & $82.7(2) \downarrow$ & $80.3(3) \downarrow$ & $1.010^{-4}(1) \uparrow$ \\
\hline & & VG & $88.3(1)^{[0.6,1]}$ & $85.5(2)^{[0.6,1]}$ & $1.610^{-4}(2)^{[1,0.6]}$ \\
\hline \multirow[t]{14}{*}{30} & \multirow[t]{2}{*}{$S_{d}^{p}$} & $\mathrm{AD}$ & $94.5(9)$ & $94.2(1)$ & $2.710^{-4}(1) \uparrow$ \\
\hline & & VG & $94.0(1)^{[1,1]}$ & $93.7(1)^{[1,1]}$ & $5.710^{-4}(9)^{[0.6,0.6]}$ \\
\hline & \multirow[t]{2}{*}{$S_{\mathrm{e}}^{p}$} & $\mathrm{AD}$ & $94.5(9)$ & $94.2(1)$ & $2.710^{-4}(1) \uparrow$ \\
\hline & & VG & $94.0(1)^{[1,1]}$ & $93.7(1)^{[1,1]}$ & $5.710^{-4}(9)^{[0.6,0.6]}$ \\
\hline & \multirow[t]{2}{*}{$S_{m}^{p}$} & $\mathrm{AD}$ & $94.5(9)$ & $94.2(1)$ & $1.410^{-4}(9) \uparrow$ \\
\hline & & VG & $94.0(1)^{[1,1]}$ & $93.7(1)^{[1,1]}$ & $2.810^{-4}(4)^{[0.6,0.6]}$ \\
\hline & \multirow[t]{2}{*}{$S_{z}$} & $\mathrm{AD}$ & $94.5(9)$ & $94.2(1)$ & $2.710^{-4}(1) \uparrow$ \\
\hline & & VG & $94.0(1)^{[1,1]}$ & $93.7(1)^{[1,1]}$ & $5.710^{-4}(9)^{[0.6,0.6]}$ \\
\hline & \multirow[t]{2}{*}{$S_{n}^{p}$} & $\mathrm{AD}$ & $94.5(9)$ & $94.2(1)$ & $5.410^{-4}(3) \uparrow$ \\
\hline & & VG & $94.0(1)^{[1,1]}$ & $93.7(1)^{[1,1]}$ & $1.110^{-3}(1)^{[0.6,0.6]}$ \\
\hline & \multirow[t]{2}{*}{$S_{C}$} & $\mathrm{AD}$ & $92.3(2) \downarrow$ & $91.8(2) \downarrow$ & $2.510^{-4}(3) \uparrow$ \\
\hline & & VG & $94.7(1)^{[0.6,1]}$ & $93.1(2)^{[0.6,1]}$ & $2.010^{-4}(2)^{[1,0.6]}$ \\
\hline & \multirow[t]{2}{*}{$S_{S}$} & $\mathrm{AD}$ & $83.6(2) \downarrow$ & $81.4(3) \downarrow$ & $7.010^{-5}(7) \uparrow$ \\
\hline & & VG & $88.7(1)^{[0.6,1]}$ & $85.7(2)^{[0.6,1]}$ & $9.010^{-5}(1)^{[1,0.6]}$ \\
\hline
\end{tabular}

A two-tailed paired $t$-test at $5 \%$ for each metric was performed. In the rows presenting the AD results of the experimental tables, $\uparrow$ or $\downarrow$ indicates that $\mathrm{AD}$ is significantly better or worse, respectively, than VG, while no mark means no significant difference. Significant differences occur mostly for assessment with AvD, especially testing BBC. Only two similarity measures, i.e., $S_{C}$ and $S_{S}$, produces significant differences for MFM and MAvG. Even though we cannot conclude which method is more efficient, the optimal results of VG are seen to depend on evaluation metrics and similarity measures.

\section{Comparison with other methods}

The proposed framework was also compared with classification by four standard models, i.e., decision tree (DT) using C4.5, naïve Bayes (NB), $k$ nearest neighbour ( $k$-NN), and support vector machine (SVM) based on the RBF kernel. The Weka machine learning suite (www.cs.waikato.ac.nz/ml/ weka) was employed for classifier learning and evaluation, using its default parameters. As observed during the learning process, 3-NN performed slightly better than $1-\mathrm{NN}, 5-\mathrm{NN}$, and $7-\mathrm{NN}$ on our 
Table 6 Comparison between the VG and AD methods on BBCSport with top $N \%$ of features ranked by GR.

\begin{tabular}{|c|c|c|c|c|c|}
\hline$N$ & Sim & $\mathrm{W}$ & MFM(SD) & MAvG(SD) & $\mathrm{AvD}(\mathrm{SD})$ \\
\hline \multirow[t]{14}{*}{50} & \multirow[t]{2}{*}{$S_{d}^{p}$} & $\mathrm{AD}$ & $93.9(1)$ & 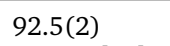 & $1.010^{-3}(3)$ \\
\hline & & VG & $93.7(6)^{[1,1]}$ & $91 \cdot 7(7)^{[1,1]}$ & $0.810^{-3}(2)^{[0.6,0.6]}$ \\
\hline & \multirow[t]{2}{*}{$S_{\mathrm{e}}^{p}$} & $\mathrm{AD}$ & $93.9(1)$ & $2.5(2)$ & $1.010^{-3}(3)$ \\
\hline & & VG & 93. & 1] & ${ }^{3}(2)^{[}$ \\
\hline & \multirow[t]{2}{*}{$S_{m}^{p}$} & $\mathrm{AD}$ & $93.9(1)$ & 5 & $0^{-3}(2)$ \\
\hline & & VG & 93. & & ${ }^{-4}(8)^{[}$ \\
\hline & \multirow[t]{2}{*}{$S_{z}$} & $\mathrm{AD}$ & 93.9 & & $0^{-3}(3)$ \\
\hline & & VG & 93. & {$[1,1]$} & $0^{-3}(2)^{[0}$ \\
\hline & \multirow[t]{2}{*}{$S_{n}^{p}$} & $\mathrm{AD}$ & 93. & & )$^{-3}(7)$ \\
\hline & & VG & 93. & & $-3(3)^{[0.6,}$ \\
\hline & \multirow[t]{2}{*}{$S_{C}$} & $\mathrm{AD}$ & 91 & $\varepsilon$ & $-4(3)$ \\
\hline & & VG & 93. & 5,1] & $0^{-4}(3)^{[1,0.6]}$ \\
\hline & \multirow[t]{2}{*}{$S_{S}$} & $\mathrm{AD}$ & 90. & 87.2( & $2.610^{-4}(3) \downarrow$ \\
\hline & & VG & & & $-4(1)^{[1,0.6]}$ \\
\hline \multirow[t]{14}{*}{80} & \multirow[t]{2}{*}{$S_{d}^{p}$} & $\mathrm{AD}$ & 93. & 0 & $0^{-3}(2)$ \\
\hline & & VG & {$[1,1]$} & {$[1,1]$} & $-3(3)^{[0.6, c}$ \\
\hline & \multirow[t]{2}{*}{$S_{\mathrm{e}}^{p}$} & $\mathrm{AD}$ & 93 & & $-3(2)$ \\
\hline & & VG & 94. & & $-3(3)^{[0.6,0.6]}$ \\
\hline & \multirow[t]{2}{*}{$S_{m}^{p}$} & $\mathrm{AD}$ & 93.7 & 922 & $0.310^{-3}(1)$ \\
\hline & & VG & & & $-4(1)^{[C}$ \\
\hline & \multirow[t]{2}{*}{$S_{z}$} & $\mathrm{AD}$ & $93.7(1$ & 92.2 & $0.710^{-3}(2)$ \\
\hline & & VG & 94. & & )$^{-4}(3)^{[0}$ \\
\hline & \multirow[t]{2}{*}{$S_{n}^{p}$} & $\mathrm{AD}$ & 93.7 & 922 & $1.510^{-3}(5)$ \\
\hline & & VG & 94. & & $0^{-3}(1)^{[0.6}$ \\
\hline & \multirow[t]{2}{*}{$S_{C}$} & $\mathrm{AD}$ & 91.60 & 88.9 & $2.310^{-4}(1) \downarrow$ \\
\hline & & VG & 92.3 & 89.9 (1 & $1.710^{-4}(1)^{[1,0.6]}$ \\
\hline & \multirow[t]{2}{*}{$S_{S}$} & $\mathrm{AD}$ & $90.9(2)$ & & $-4(2) \downarrow$ \\
\hline & & VC & $88.4 \mathrm{C}$ & 83.5 & $-4(1)^{[1,0.6]}$ \\
\hline
\end{tabular}

training data sets, and was chosen as a representative of $k$-NN. The 5 -fold CV with the same separate datasets used in the previous section was used for evaluating the four models.

Table 8 compares our framework with the four classification models when used on BBC. The first two columns detail a method for calculating relevant scores of feature words and the numbers of selected features. The next column expresses a similarity measure or a classification model in use. The last two columns show evaluation results in terms of MFM and MAvG including standard deviation. Since all distance-based similarity measures give the same values of MFM and MAvG, only results of $S_{m}^{p}$ are shown in this table. Note that, even if the memory was extended, Weka ran out of memory in the training process of SVM in both cases of selecting $30 \%$ of features. Hence there is no report for these cases. The table indicates that, regardless of similarity measures, our framework yields much higher performance than DT and $k$-NN. Using top 30\% of features rating by GR, for example, $S_{m}^{p}$ gives $95 \%$ and $94 \%$ of MFM and MAvG, respectively, while $k$ NN produces $52 \%$ and $44 \%$. Comparing with NB
Table 7 Comparison between the VG and AD methods on BBCSport with top $N \%$ of features ranked by IG.

\begin{tabular}{|c|c|c|c|c|c|}
\hline$N$ & Sim & $\mathrm{W}$ & MFM(SD) & MAvG(SD) & $\mathrm{AvD}(\mathrm{SD})$ \\
\hline \multirow[t]{14}{*}{50} & \multirow[t]{2}{*}{$S_{d}^{p}$} & $\mathrm{AD}$ & $93.9(1)$ & $92.4(2)$ & $1.010^{-3}(3)$ \\
\hline & & VG & $93.8(6)^{[1,1]}$ & $91.9(9)^{[1,1]}$ & $8.110^{-4}(9)^{[0.6,0.6]}$ \\
\hline & \multirow[t]{2}{*}{$S_{\mathrm{e}}^{p}$} & $\mathrm{AD}$ & $93.9(1)$ & $92.4(2)$ & $1.010^{-3}(4)$ \\
\hline & & VG & $93.8(6)^{[1,1]}$ & $91.9(9)^{[1,1]}$ & $8.110^{-4}(9)^{[0.6,0.6]}$ \\
\hline & \multirow[t]{2}{*}{$S_{m}^{p}$} & $\mathrm{AD}$ & $93.9(1)$ & $92.4(2)$ & $0.510^{-3}(2)$ \\
\hline & & VG & $93.8(6)^{[1,1]}$ & $91.9(9)^{[1,1]}$ & $4.010^{-4}(4)^{[0.6,0.6]}$ \\
\hline & \multirow[t]{2}{*}{$S_{z}$} & $\mathrm{AD}$ & $93.9(1)$ & $92.4(2)$ & $1.010^{-3}(3)$ \\
\hline & & VG & $93.8(6)^{[1,1]}$ & $91.9(9)^{[1,1]}$ & $8.110^{-4}(9)^{[0.6,0.6]}$ \\
\hline & \multirow[t]{2}{*}{$S_{n}^{p}$} & $\mathrm{AD}$ & $93.9(1)$ & $92.4(2)$ & $2.110^{-3}(6)$ \\
\hline & & VG & $93.8(6)^{[1,1]}$ & $91.9(9)^{[1,1]}$ & $1.610^{-3}(1)^{[0.6,0.6]}$ \\
\hline & \multirow[t]{2}{*}{$S_{C}$} & $\mathrm{AD}$ & $91.1(9)$ & $88.1(1)$ & $3.410^{-4}(5) \downarrow$ \\
\hline & & VG & $93.7(1)^{[0.6,1]}$ & $89.0(1)^{[0.6,1]}$ & $2.610^{-4}(4)^{[1,0.6]}$ \\
\hline & \multirow[t]{2}{*}{$S_{S}$} & $\mathrm{AD}$ & $90.4(1) \uparrow$ & $87.2(2) \uparrow$ & $2.610^{-4}(3) \downarrow$ \\
\hline & & VG & $87.6(1)^{[1,0.6]}$ & $82.6(2)^{[1}$ & $1.110^{-4}(1)^{[1,0.6]}$ \\
\hline \multirow[t]{14}{*}{80} & \multirow[t]{2}{*}{$S_{d}^{p}$} & $\mathrm{AD}$ & $93.9(1)$ & $92.4(2)$ & $0.810^{-3}(2)$ \\
\hline & & VG & $94.1(4)^{[1,1]}$ & $92.2(6)^{[1,1]}$ & $5.810^{-4}(4)^{[0.6,0.6]}$ \\
\hline & \multirow[t]{2}{*}{$S_{\mathrm{e}}^{p}$} & $\mathrm{AD}$ & $93.9(1)$ & $92.4(2)$ & $0.810^{-3}(2)$ \\
\hline & & VG & $94.1(4)^{[1,1]}$ & $92.2(6)^{[1,1]}$ & $5.810^{-4}(4)^{[0.6,0.6]}$ \\
\hline & \multirow[t]{2}{*}{$S_{m}^{p}$} & $\mathrm{AD}$ & $93.9(1)$ & $92.4(2)$ & $0.410^{-3}(1)$ \\
\hline & & VG & $94.1(4)^{[1,1]}$ & $92.4(6)^{[1,1]}$ & $2.910^{-4}(2)^{[0.6,0.6]}$ \\
\hline & \multirow[t]{2}{*}{$S_{z}$} & $\mathrm{AD}$ & $93.9(1)$ & $92.4(2)$ & $0.810^{-3}(2)$ \\
\hline & & VG & $94.1(4)^{[1,1]}$ & $92.2(6)^{[1,1]}$ & $5.810^{-4}(5)^{[0.6,0.6]}$ \\
\hline & \multirow[t]{2}{*}{$S_{n}^{p}$} & $\mathrm{AD}$ & $93.9(1)$ & $92.4(2)$ & $1.610^{-3}(5)$ \\
\hline & & VG & $94.1(4)^{[1,1]}$ & $92.2(2)^{[1,1]}$ & $1.110^{-3}(9)^{[0.6,0.6]}$ \\
\hline & \multirow[t]{2}{*}{$S_{C}$} & $\mathrm{AD}$ & $91.6(1)$ & $88.9(1)$ & $2.310^{-4}(1) \downarrow$ \\
\hline & & VG & $92.4(6)^{[0.6,1]}$ & $89.9(1)^{[0.6,1]}$ & $1.810^{-4}(1)^{[1,0.6]}$ \\
\hline & \multirow[t]{2}{*}{$S_{S}$} & $\mathrm{AD}$ & $91.0(2)$ & $88.3(3) \uparrow$ & $1.810^{-4}(2) \downarrow$ \\
\hline & & VG & $88.4(1)^{[1,0.6]}$ & $82.5(2)^{[1,0.6]}$ & $7.010^{-5}(7)^{[1,0.6]}$ \\
\hline
\end{tabular}

and SVM, the table reveals that the performances of $S_{m}^{p}$ and $S_{C}$ are close to those of NB and SVM, while those of $S_{S}$ are worse.

A paired $t$-test at $\alpha=5 \%$ was performed to assess the statistical significance of the difference between our framework with a particular measure and one of the four classifiers. The comparison result of $\uparrow,-$, or $\downarrow$ means that the measure is statistically superior, equal, or inferior to the classifier. The order of classifiers to be compared is DT, $k$ $\mathrm{NN}, \mathrm{NB}$, and SVM. For instance, the comparison result of MFM values obtained from $S_{C}$ to those from the classifiers reported in the 15th row of Table 8 is $[\uparrow, \uparrow,-, \downarrow]$. It means that $S_{C}$ is significantly better than DT and $k-\mathrm{NN}$, equal to NB, and worse than SVM. Among the 4 cases of feature selection, $S_{m}^{p}$ and $S_{C}$ are statistically better than DT and $k$-NN when they gain the comparative performance to NB, and SVM, except the third case (i.e., $20 \%$ of features ranked by IG) where $S_{C}$ is significantly worse than SVM. For $S_{S}$, it yields a higher accuracy than DT and $k$-NN, but lower than NB and SVM.

Likewise, Table 9 presents results evaluating BBCSport. It indicates that, no matter what simi- 
Table 8 Comparison with other classifiers when evaluating BBC.

\begin{tabular}{|c|c|c|c|c|}
\hline $\begin{array}{l}\text { Feature } \\
\text { selection }\end{array}$ & $N$ & $\begin{array}{l}\text { Sim/ } \\
\text { Classifier }\end{array}$ & MFM(SD) & MAvG(SD) \\
\hline \multirow[t]{7}{*}{ GR } & \multirow[t]{7}{*}{20} & $S_{m}^{p}$ & $93.2(4)^{[\uparrow, \uparrow,-,-]}$ & $92.6(4)^{[\uparrow, \uparrow,-,-]}$ \\
\hline & & $S_{C}$ & $92.8(1)^{[\uparrow, \uparrow,-,-]}$ & $92.1(2)^{[\uparrow, \uparrow,-,-]}$ \\
\hline & & $S_{S}$ & $85.1(2)^{[\uparrow, \uparrow, \downarrow, \downarrow]}$ & $82.4(2)^{[\uparrow, \uparrow, \downarrow, \downarrow, \downarrow]}$ \\
\hline & & DT & $81.2(3)$ & $81.0(3)$ \\
\hline & & $k-\mathrm{NN}$ & $63.3(3)$ & $58.2(2)$ \\
\hline & & NB & $93.7(6)$ & $93.8(6)$ \\
\hline & & SVM & $93.4(7)$ & $93.5(8)$ \\
\hline \multirow[t]{6}{*}{ GR } & \multirow[t]{6}{*}{30} & $S_{m}^{p}$ & $94.7(6)^{[\uparrow, \uparrow,-]}$ & $94.4(7)^{[\uparrow, \uparrow,-]}$ \\
\hline & & $S_{C}$ & $93.4(1)^{[\uparrow, \uparrow,-]}$ & $93.0(1)^{[\uparrow, \uparrow,-]}$ \\
\hline & & $S_{S}$ & $84.8(2)^{[\uparrow, \uparrow, \downarrow]}$ & $82.8(2)^{[\uparrow, \uparrow, \downarrow]}$ \\
\hline & & DT & $69.1(2)$ & $68.4(2)$ \\
\hline & & $k-\mathrm{NN}$ & $52.3(7)$ & $44.0(6)$ \\
\hline & & NB & $93.9(1)$ & $94.0(1)$ \\
\hline \multirow[t]{7}{*}{ IG } & \multirow[t]{7}{*}{20} & $S_{m}^{p}$ & $94.4(9)^{[\uparrow, \uparrow,-,-]}$ & $94.0(1)^{[\uparrow, \uparrow,-,-]}$ \\
\hline & & $S_{C}$ & $92.3(2)^{[\uparrow, \uparrow,-, \downarrow]}$ & $91.8(2)^{[\uparrow, \uparrow,-, \downarrow]}$ \\
\hline & & $S_{S}$ & $82.7(2)^{[\uparrow, \uparrow, \downarrow, \downarrow]}$ & $80.3(3)^{[\uparrow, \uparrow, \downarrow, \downarrow]}$ \\
\hline & & DT & $69.8(3)$ & $69.4(3)$ \\
\hline & & $k-\mathrm{NN}$ & $60.2(4)$ & $53.2(4)$ \\
\hline & & NB & $93.9(9)$ & $93.9(9)$ \\
\hline & & SVM & $94.4(1)$ & $94.5(2)$ \\
\hline \multirow[t]{6}{*}{ IG } & \multirow[t]{6}{*}{30} & $S_{m}^{p}$ & $94.5(9)^{[\uparrow, \uparrow,-]}$ & $94.2(1)^{[\uparrow, \uparrow,-]}$ \\
\hline & & $S_{C}$ & $92.3(2)^{[\uparrow, \uparrow,-]}$ & $91.8(2)^{[\uparrow, \uparrow,-]}$ \\
\hline & & $S_{S}$ & $83.6(2)^{[\uparrow, \uparrow, \downarrow]}$ & $81.4(3)^{[\uparrow, \uparrow, \downarrow]}$ \\
\hline & & DT & $66.5(3)$ & $65.7(3)$ \\
\hline & & $k-\mathrm{NN}$ & $49.8(3)$ & $41.2(3)$ \\
\hline & & NB & $94.1(8)$ & $94.2(9)$ \\
\hline
\end{tabular}

larity measure was used, the proposed framework clearly outperforms DT and $k$-NN. $S_{m}^{p}$ shows comparable performance to NB and SVM. $S_{C}$ is worse than NB, while $S_{S}$ is worse than both NB and SVM.

\section{CONCLUSIONS}

With statistical concepts, documents can be represented in terms of IFSs and patterns for predefined document classes can be constructed. A similarity measure is used to determine a similarity degree between an IFS for a document and a class pattern. The document is grouped into the class such that its pattern is closest to the IFS for the document. Using some existing similarity measures for IFSs, the experiment on two datasets shows that our framework yields satisfactory results when compared to results from traditional classification models. Further work includes extension of the dataset, investigation of various techniques for IFS-based document representation, and in-depth analysis of IFS to facilitate text classification.
Table 9 Comparison with other classifiers when evaluating BBCSport.

\begin{tabular}{|c|c|c|c|c|}
\hline $\begin{array}{l}\text { Feature } \\
\text { selection }\end{array}$ & $N$ & $\begin{array}{l}\text { Sim/ } \\
\text { Classifier }\end{array}$ & MFM(SD) & MAvG(SD) \\
\hline \multirow[t]{7}{*}{ GR } & \multirow[t]{7}{*}{50} & $S_{m}^{p}$ & $93.9(1)^{[\uparrow, \uparrow,-, \uparrow]}$ & $92.5(2)^{[\uparrow, \uparrow, \downarrow,-]}$ \\
\hline & & $S_{C}$ & $91.3(6)^{[\uparrow, \uparrow, \downarrow,-]}$ & $88.4(1)^{[\uparrow, \uparrow, \downarrow,-]}$ \\
\hline & & $S_{S}$ & $90.2(1)^{[\uparrow, \uparrow, \downarrow,-]}$ & $87.2(2)^{[\uparrow, \uparrow, \downarrow, \downarrow]}$ \\
\hline & & DT & $70.4(4)$ & $69.9(3)$ \\
\hline & & $k-\mathrm{NN}$ & $41.5(6)$ & $30.1(6)$ \\
\hline & & NB & $96.1(1)$ & $96.2(1)$ \\
\hline & & SVM & $89.8(4)$ & $91.3(3)$ \\
\hline \multirow[t]{7}{*}{ GR } & \multirow[t]{7}{*}{80} & $S_{m}^{p}$ & $93.7(1)^{[\uparrow, \uparrow,-,-]}$ & $92.2(2)^{[\uparrow, \uparrow,-,-]}$ \\
\hline & & $S_{C}$ & $91.6(1)^{[\uparrow, \uparrow, \downarrow,-]}$ & $88.9(2)^{[\uparrow, \uparrow, \downarrow,-]}$ \\
\hline & & $S_{S}$ & $90.9(2)^{[\uparrow, \uparrow, \downarrow,-]}$ & $88.1(3)^{[\uparrow, \uparrow,-,-]}$ \\
\hline & & DT & $63.4(4)$ & $61.6(4)$ \\
\hline & & $k-\mathrm{NN}$ & $41.8(6)$ & $30.6(7)$ \\
\hline & & NB & $95.3(2)$ & $95.7(2)$ \\
\hline & & SVM & $89.8(45)$ & $91.0(4)$ \\
\hline \multirow[t]{7}{*}{ IG } & \multirow[t]{7}{*}{50} & $S_{m}^{p}$ & $93.9(1)^{[\uparrow, \uparrow,-,-]}$ & $92.4(2)^{[\uparrow, \uparrow, \downarrow,-]}$ \\
\hline & & $S_{C}$ & $91.1(9)^{[\uparrow, \uparrow, \downarrow,-]}$ & $88.1(1)^{[\uparrow, \uparrow, \downarrow,-]}$ \\
\hline & & $S_{S}$ & $90.4(1)^{[\uparrow, \uparrow, \downarrow,-]}$ & $87.2(2)^{[\uparrow, \uparrow, \downarrow,-]}$ \\
\hline & & DT & $69.9(4)$ & $68.9(4)$ \\
\hline & & $k-\mathrm{NN}$ & $42.2(9)$ & $30.2(9)$ \\
\hline & & NB & $95.9(1)$ & $96.3(2)$ \\
\hline & & SVM & $89.4(4)$ & $90.9(4)$ \\
\hline \multirow[t]{7}{*}{ IG } & \multirow[t]{7}{*}{80} & $S_{m}^{p}$ & $93.9(1)^{[\uparrow, \uparrow,-,-]}$ & $92.4(2)^{[\uparrow, \uparrow,-,-]}$ \\
\hline & & $S_{C}$ & $91.6(1)^{[\uparrow, \uparrow, \downarrow,-]}$ & $88.9(2)^{[\uparrow, \uparrow, \downarrow,-]}$ \\
\hline & & $S_{S}$ & $91.0(2)^{[\uparrow, \uparrow, \downarrow,-]}$ & $88.3(3)^{[\uparrow, \uparrow, \downarrow, \downarrow]}$ \\
\hline & & DT & $66.6(5)$ & $65.5(5)$ \\
\hline & & $k-\mathrm{NN}$ & $40.4(2)$ & $28.6(1)$ \\
\hline & & NB & $95.5(2)$ & $95.8(2)$ \\
\hline & & SVM & $90.1(4)$ & $91.2(4)$ \\
\hline
\end{tabular}

Acknowledgements: The author gratefully acknowledges the financial support provided by Thammasat University Research Fund under the TU Research Scholar scheme, Contract No. 1/8/2557.

\section{REFERENCES}

1. Zadeh LA (1965) Fuzzy sets. Inform Contr 8, 338-53.

2. Atanassov K (1986) Intuitionistic fuzzy sets. Fuzzy Set Syst 20, 87-96.

3. Dengfeng L, Chuntian C (2002) New similarity measures of intuitionistic fuzzy sets and application to pattern recognition. Pattern Recogn Lett 23, 221-5.

4. Liang Z, Shi P (2003) Similarity measures on intuitionistic fuzzy sets. Pattern Recogn Lett 24, 2687-93.

5. Mitchell HB (2003) On the Dengfeng-Chuntian similarity measure and its application to pattern recognition. Pattern Recogn Lett 24, 3101-4.

6. Hung WL, Yang MS (2004) Similarity measures of intuitionistic fuzzy sets based on Hausdorff distance. Pattern Recogn Lett 25, 1603-11. 
7. Xu Z (2007) Some similarity measures of intuitionistic fuzzy sets and their applications to multiple attribute decision making. Fuzzy Optim Decis Making 6, 109-21.

8. Khatibi V, Montazer GA (2009) Intuitionistic fuzzy set vs. fuzzy set application in medical pattern recognition. Artif Intell Med 47, 43-52.

9. Ye J (2011) Cosine similarity measures for intuitionistic fuzzy sets and their applications. Math Comput Model 53, 91-7.

10. Hwang CM, Yang MS (2012) Modified cosine similarity measure between intuitionistic fuzzy sets. In: 4th International Conference, AICI 2012, Chengdu, China, pp 285-93.

11. Li Y, Olson D, Qin Z (2007) Similarity measures between intuitionistic fuzzy (vague) sets: A comparative analysis. Pattern Recogn Lett 28, 278-85.

12. Papakostas GA, Hatzimichailidis AG, Kaburlasos VG (2013) Distance and similarity measures between intuitionistic fuzzy sets: A comparative analysis from a pattern recognition point of view. Pattern Recogn Lett 34, 1609-22.

13. Sebastiani F (2002) Machine learning in automated text categorization. ACM Comput Surv 34, 1-47.

14. Khan A, Baharudin B, Lee LH, Khan K (2010) A review of machine learning algorithms for textdocuments classification. $J$ Adv Inform Tech 1, 4-20.

15. Mita KD, Mukesh AZ (2012) Automatic text classification: a technical review. Int J Comput Sci Appl $\mathbf{2 8}$ $37-40$.

16. Szmidt E, Kacprzyk J (2008) Using Intuitionistic Fuzzy Sets in Text Categorization. In: 9th International Conference on Artificial Intelligence and Soft Computing, Zakopane, Poland, pp 351-62.

17. Julian P, Hung KC, Lin SJ (2012) On the Mitchell similarity measure and its application to pattern recognition. Pattern Recogn Lett 33, 1219-23.

18. Greene D, Cunningham P (2005) Producing accurate interpretable clusters from high-dimensional data. In: 9th European Conference on Principles and Practice of Knowledge Discovery in Databases, pp 486-94.

19. Sun Y, Kamel MS, Wang Y (2006) Boosting for learning multiple classes with imbalanced class distribution. In: 6th International Conference on Data Mining, pp 592-602. 